\title{
Dynamics of the Garnet/Li Interface for Dendrite-Free Solid-State Batteries
}

Hanyu Huo ${ }^{a, b}$, Jianneng Liang ${ }^{a}$, Ning Zhao ${ }^{c}$, Xiaona Li ${ }^{a}$, Xiaoting Lin ${ }^{a}$, Yang Zhao ${ }^{a}$, Keegan Adair ${ }^{a}$, Ruying Li ${ }^{a}$, Xiangxin Guo ${ }^{c *}$, Xueliang Sun ${ }^{a}{ }^{*}$

${ }^{a}$ Department of Mechanical and Materials Engineering, University of Western

Ontario, Ontario N6A 5B9, Canada

${ }^{\mathrm{b}}$ State Key Laboratory of High Performance Ceramics and Superfine Microstructure, Shanghai Institute of Ceramics, Chinese Academy of Sciences, Shanghai 200050,

China

${ }^{\mathrm{c}}$ College of Physics, Qingdao University, Qingdao 266071, China

* Corresponding authors.

Email address:xxguo@qdu.edu.cn,xsun9@uwo.ca 


\section{Experimental}

\section{Fabrication of 3D-LLZTO}

Tantalum (Ta)-doped $\mathrm{Li}_{6.4} \mathrm{La}_{3} \mathrm{Zr}_{1.4} \mathrm{Ta}_{0.6} \mathrm{O}_{12}$ (LLZTO) powders and dense pellets were prepared according to our previous work ${ }^{1}$. $1 \mathrm{M}$ hydrochloric acid $(\mathrm{HCl})$ was obtained by concentrated $\mathrm{HCl}(12 \mathrm{M})$ adding certain water. The LLZTO pellets were immersed in the $\mathrm{HCl}$ solution for $1 \mathrm{~h}$. After that, the acid-treated pellets were rapidly washed by ethanol and dried by a hairdryer. Various acid-etching time was used to examine the effect on the thickness of porous structure.

\section{Fabrication of 3D-LLZTO@ZnO}

A layer of $\mathrm{ZnO}$ with $20 \mathrm{~nm}$ thickness was coated on the 3D-LLZTO surface by the atomic layer deposition (ALD) method with Gemstar-8 ALD system (Arradiance, USA). The ALD process with 150 cycles was operated in a vacuum condition and at $150^{\circ} \mathrm{C}$. Ar gas functions as the carrier gas in the whole process. Precursors diethyl zinc (DEZ) and water were used as precursors for the ALD process. The same $\mathrm{ZnO}$ layer was also coated on the LLZTO surface for comparison.

\section{Materials characterizations}

Crystal structures of samples were examined by X-ray diffraction (XRD, Bruker D2 Phaser), using $\mathrm{Cu} \mathrm{K \alpha}$ radiation with $2 \theta$ in the range of $10^{\circ} \sim 80^{\circ}$ and a step size of $0.02^{\circ}$. Surface and cross-section morphologies of the LLZTO pellets were investigated by scanning electron microscopy (SEM, S3400 and S4800). X-ray photoelectron spectroscopy (XPS, ESCALAB-250) were performed to characterize the composition of LLZTO pellets before and after acid-etching. 


\section{Electrochemical performance tests}

Ionic conductivities of the various LLZTO samples were measured by an impedance analyzer (Novocontrol Beta High Performance Impedance Analyzer) with an $\mathrm{AC}$ of $10 \mathrm{mV}$ from 0.1 to $7 \mathrm{M} \mathrm{Hz}$ in frequency. Thin gold layers on both surfaces of ceramic pellets were performed by magnetic sputtering as electrodes before the conductivity test. The 3D-LLZTO@ZnO pellets were sandwiched between two pieces of Li metal to construct the symmetric cells. Li metal electrodes were melted onto the two sides of the LLZTO pellets at $300{ }^{\circ} \mathrm{C}$ for $30 \mathrm{~min}$ in an Ar-filled glovebox before sealing in Swagelok-type cell mold. A pressure of approximately $10 \mathrm{~N} \mathrm{~cm}^{-2}$ was exerted on the ceramic plates via springs to keep close contact. Electrochemical impedance spectroscopy (EIS) measurements were performed in the frequency range from $1 \mathrm{MHz}$ to $0.1 \mathrm{~Hz}$ with an amplitude of $10 \mathrm{mV}$ by an Autolab instrument. Galvanostatic cycling tests were conducted using a Land battery cycler under different current densities at 25 ${ }^{\circ} \mathrm{C}$. Li/LLZTO/Li and Li/LLZTO@ZnO/Li symmetric cells were also fabricated and cycled under the same procedure as a comparison. Li metal anodes with two different thickness $(200 \mu \mathrm{m}$ and $30 \mu \mathrm{m})$ were used for investigating the effect of Li volume change on the Li dendrite growth. The Li anode was the $\sim 200 \mu \mathrm{m} \mathrm{Li} \mathrm{without} \mathrm{any} \mathrm{special}$ notification. Three cells were assembled for cross-sections at different states during plating and stripping at $0.5 \mathrm{~mA} \mathrm{~cm}^{-2}\left(1 \mathrm{mAh} \mathrm{cm}^{-2}\right)$.

To fabricate "all-in-one" LFP/Li cells, the LFP, PVDF, LiTFSI, and SP with the weight ratio of LFP: LiTFSI: SP: PVDF=5: 3.5: 1: 0.5 were mixed together to fabricate cathode slurry using N-Methyl pyrrolidone (NMP) as solvent ${ }^{2}$. The slurry was 
infiltrated into the LLZTO framework and heated at $80{ }^{\circ} \mathrm{C}$ in a vacuum oven for $12 \mathrm{~h}$. The LFP loading is $\sim 2 \mathrm{mg} \mathrm{cm}^{-2}$ and $1 \mathrm{C}=170 \mathrm{mAh} \mathrm{g}^{-1}$. The $\sim 30 \mu \mathrm{m}$-thick Li was infiltrated into the other side of the LLZTO porous framework. The LFP cathodes with a similar LFP loading were also coated on LLZTO and LLZTO@ZnO pellets to fabricate LFP/Li full cells for comparison. The LFP/Li cells were operated at $60{ }^{\circ} \mathrm{C}$. The GITT was carried out with a pulse current of $0.1 \mathrm{C}$ for $10 \mathrm{~min}$ and rest for $30 \mathrm{~min}$ at the $2^{\text {nd }}$ cell cycle.

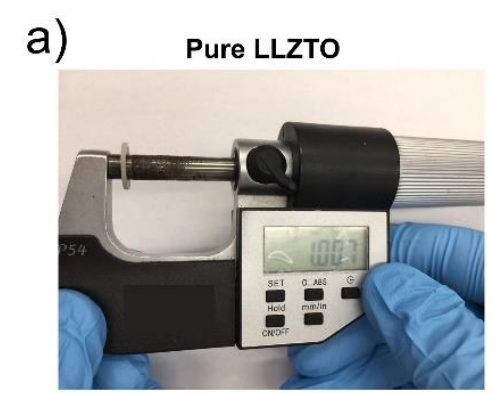

$\mathrm{D}=1.007 \mathrm{~mm}$

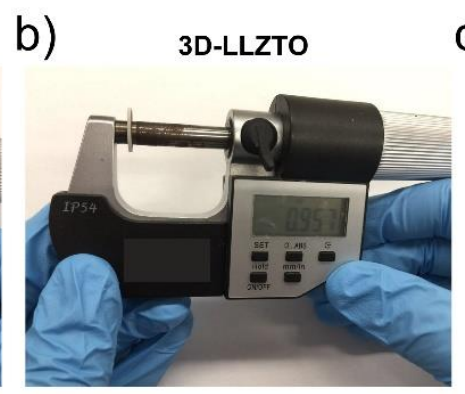

$\mathrm{D}=0.957 \mathrm{~mm}$
C) 3D-LLZTO after sanding

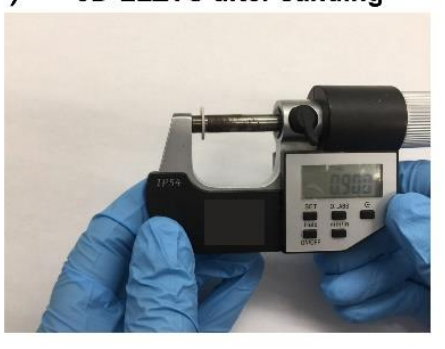

$\mathrm{D}=0.900 \mathrm{~mm}$

Figure S1 Thickness measurements of a) pure LLZTO, b) 3D-LLZTO by acid-etching and c) 3D-LLZTO after sanding the upper 3D layers. 

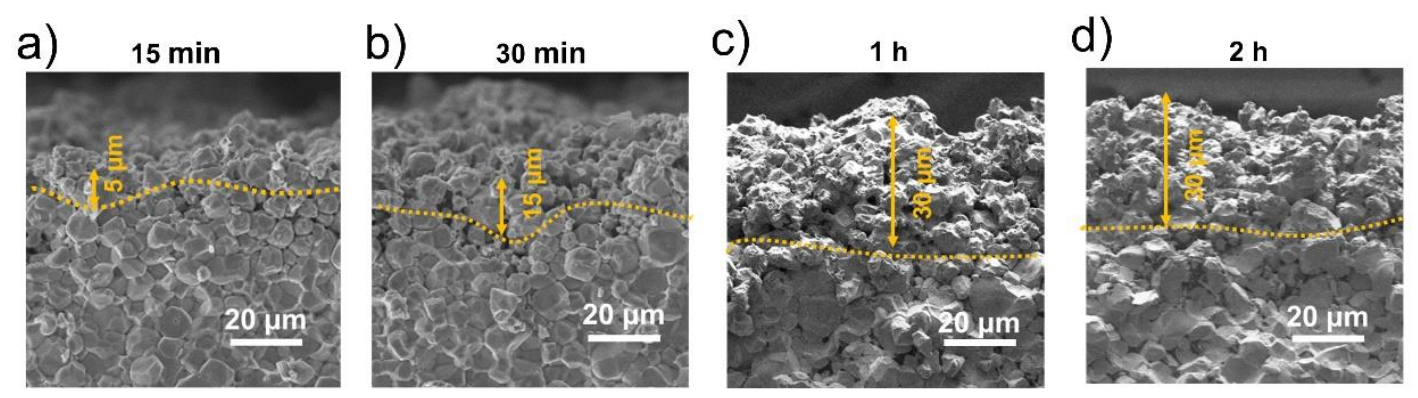

Figure S2 Cross-sectional SEM images of acid-etched 3D-LLZTO for a) $15 \mathrm{~min}, \mathrm{~b}) 30$ $\min , \mathrm{c}) 1 \mathrm{~h}$, and d) $2 \mathrm{~h}$.

As shown in Figure S2, the thickness of the porous layer increases from $5 \mu \mathrm{m}$ to $30 \mu \mathrm{m}$ when the time for acid etching increases from $15 \mathrm{~min}$ to $1 \mathrm{~h}$. Further increasing the etching time cannot increase the thickness of the 3D structure, indicating a dynamic equilibrium after $1 \mathrm{~h}$ acid etching. In order to obtain various thicknesses and porosities of 3D LLZTO frameworks, the concentration of $\mathrm{HCl}$ can be changed and some surfaceactive agents can be added in the $\mathrm{HCl}$ solution, which will be studied in the future. 


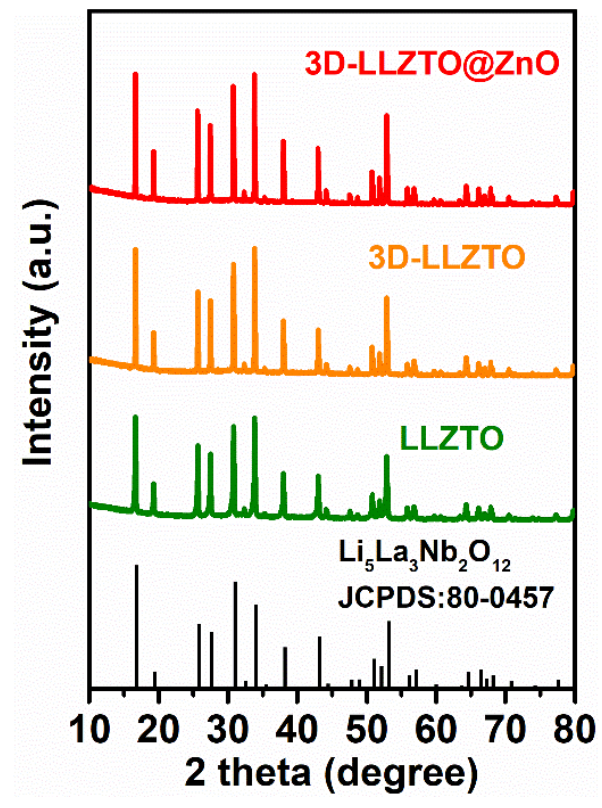

Figure S3 XRD patterns of LLZTO, 3D-LLZTO and 3D-LLZTO@ZnO. 

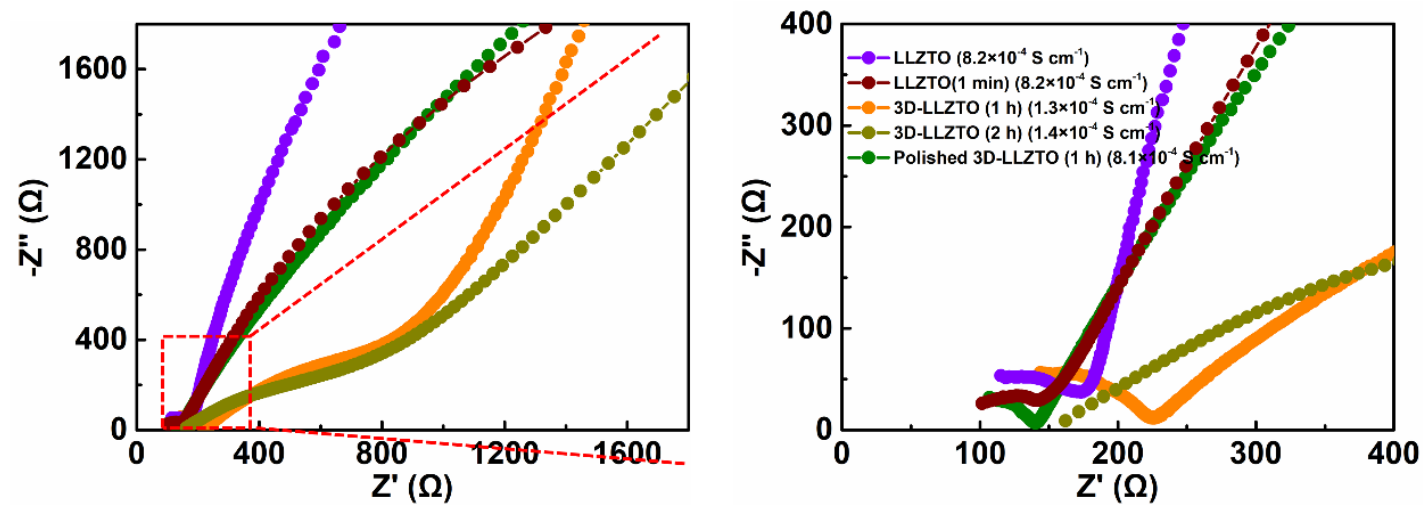

Figure S4 EIS spectra of LLZTO, LLZTO (1 min), 3D-LLZTO (1 h), 3D-LLZTO (2 h), and polished 3D-LLZTO (1 h). 


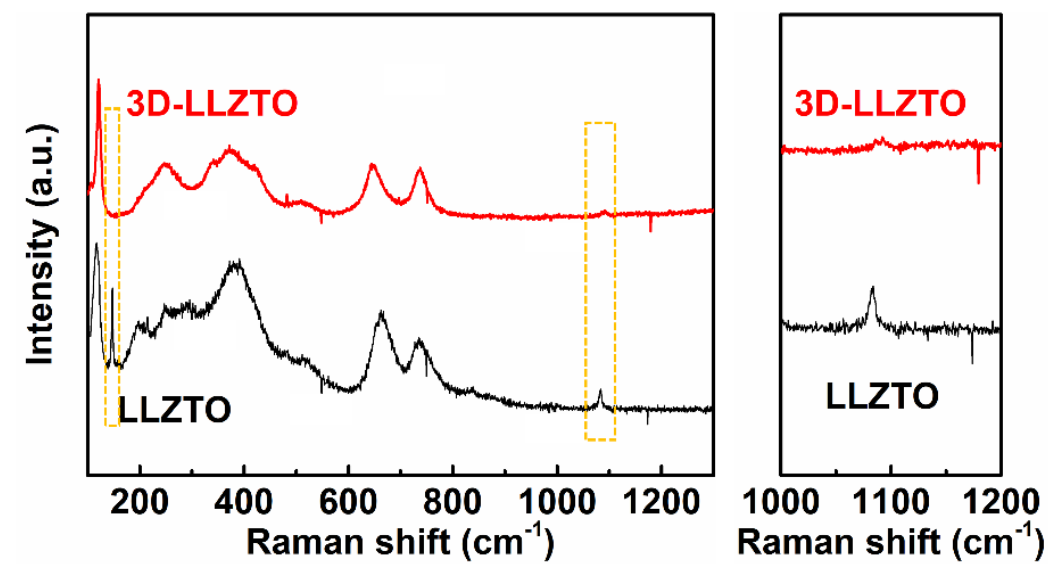

Figure S5 Raman spectra of the LLZTO and 3D-LLZTO by acid etching. 

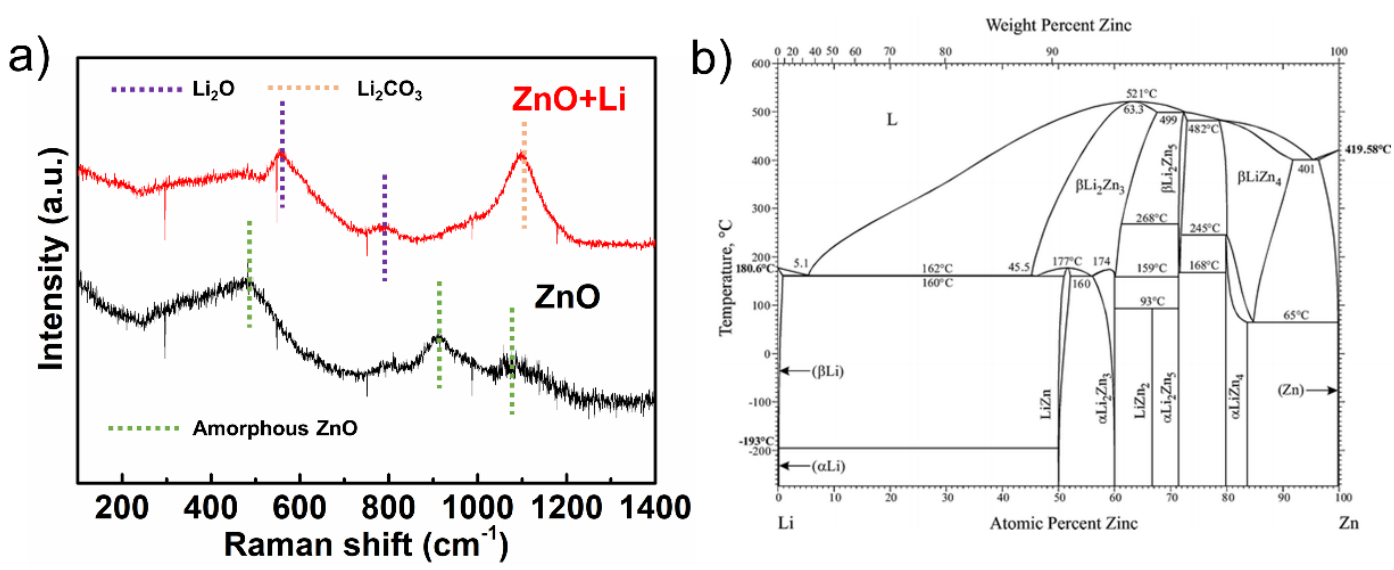

Figure $\mathrm{S} 6$ a) Raman spectra of the $\mathrm{ZnO}$ thin film on the glass plate and $\mathrm{ZnO}$ thin film contacted with molten Li on the glass plate. b) $\mathrm{Zn}-\mathrm{Li}$ alloy phase diagram ${ }^{3}$.

The interfacial contact can be greatly improved by the $\mathrm{ZnO}$ layer. We further characterized the reactions at the interface by Raman spectra. As shown in Fig. 4a, the peaks at 497, 908, and $1086 \mathrm{~cm}^{-1}$ correspond to the amorphous $\mathrm{ZnO}$ by ALD ${ }^{4}$. These peaks of $\mathrm{ZnO}$ disappear and the $\mathrm{Li}_{2} \mathrm{O}$ peaks at 521 and $796 \mathrm{~cm}^{-1}$ are obtained when the $\mathrm{ZnO}$ is contacted with the molten $\mathrm{Li}$ at $300{ }^{\circ} \mathrm{C}$. The enhanced wettability at the interface could be attributed to the conversion reaction between $\mathrm{ZnO}$ and $\mathrm{Li}$ (equation (1)).

$$
\mathrm{ZnO}(\mathrm{s})+\mathrm{Li}(\mathrm{l}) \rightarrow \mathrm{Li}_{2} \mathrm{O}(\mathrm{s})+\mathrm{Zn}(\mathrm{s})
$$

Subsequently, the $\mathrm{Zn}$ further reacts with the Li to form alloy at $300{ }^{\circ} \mathrm{C}$ according to the $\mathrm{Zn}$-Li alloy phase diagram (equation (2)). The as-formed $\mathrm{Zn}-\mathrm{Li}$ alloy shows the excellent ion/electron conductivity, which can guide uniform Li deposition. It should be noted that the $\mathrm{Li}_{2} \mathrm{CO}_{3}$ peak at $1090 \mathrm{~cm}^{-1}$ could be attributed to the briefly exposed Li metal to air during the sample transfer for Raman testing.

$$
\mathrm{Zn}(\mathrm{s})+\operatorname{Li}(\mathrm{l}) \rightarrow \operatorname{LiZn}(\mathrm{s})
$$




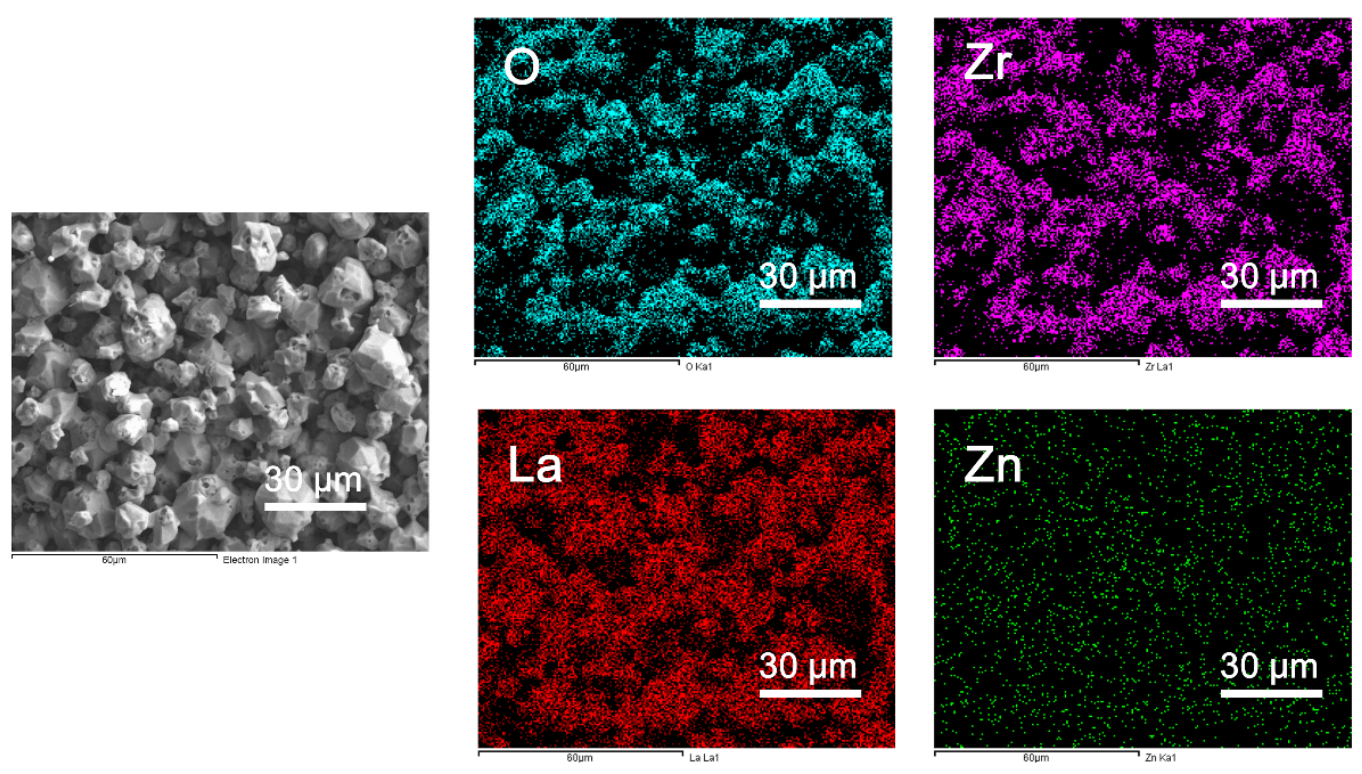

Figure S7 Top-view SEM images and EDS mappings of the 3D-LLZTO@ZnO pellet. 
a)

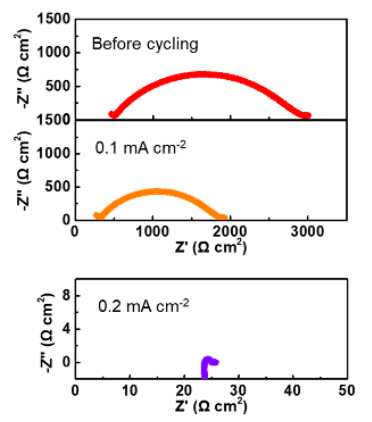

b)

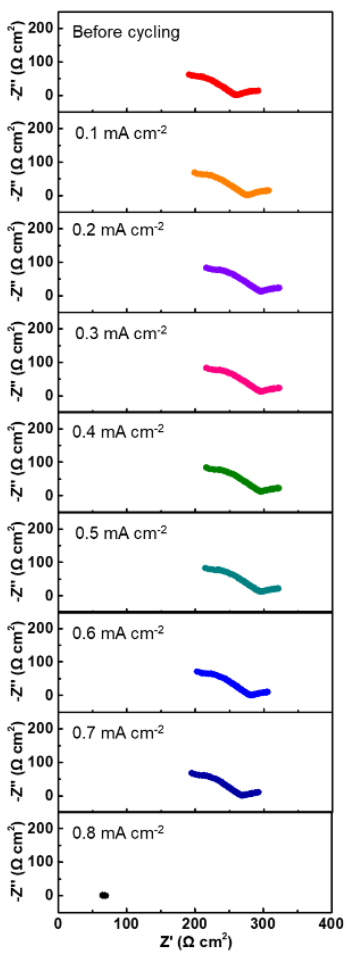

c)

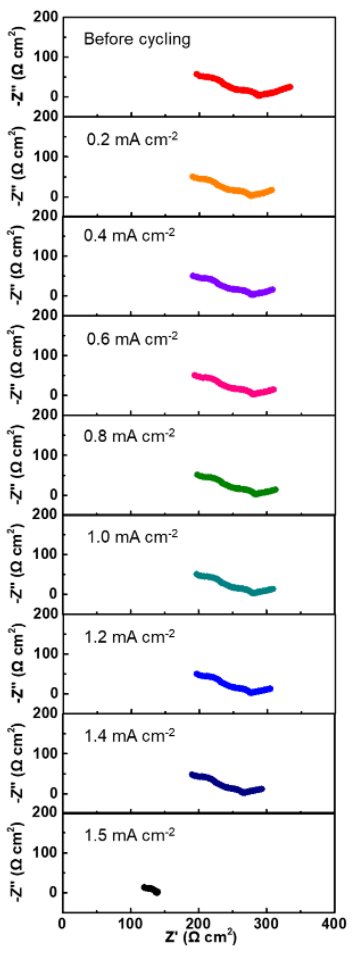

Figure S8 EIS spectra of a) Li/LLZTO/Li, b) Li/LLZTO@ZnO/Li, and c) Li/3DLLZTO@ZnO/Li cells during CCD tests. 

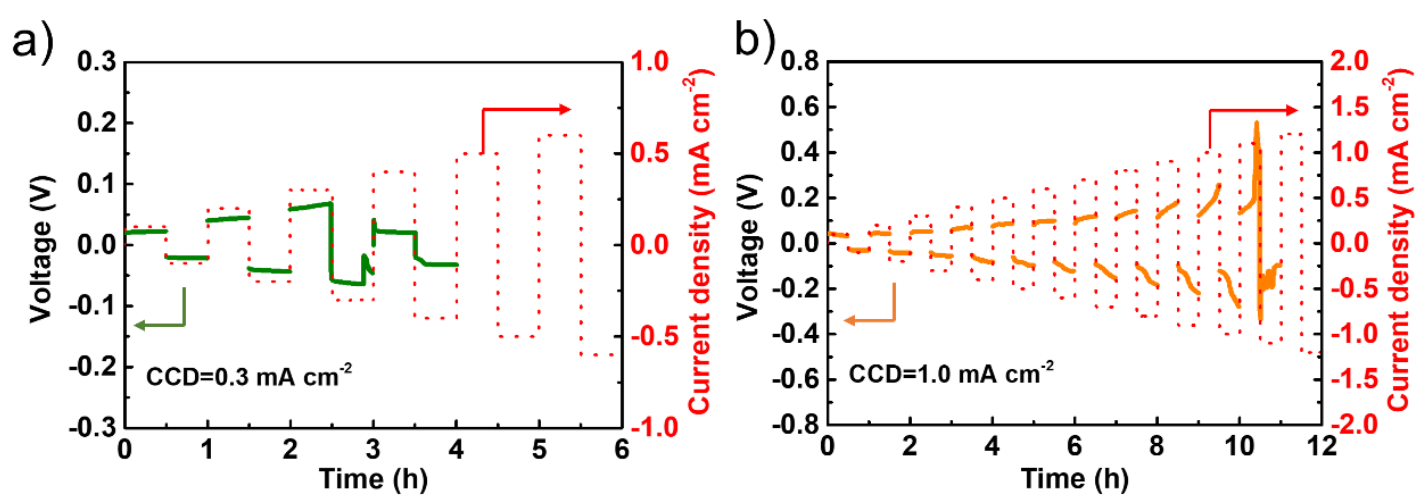

Figure S9 CCD of a) Li/LLZTO@ZnO/Li, and b) Li/3D-LLZTO@ZnO/Li cells with thin Li anodes $(\sim 30 \mu \mathrm{m})$. 

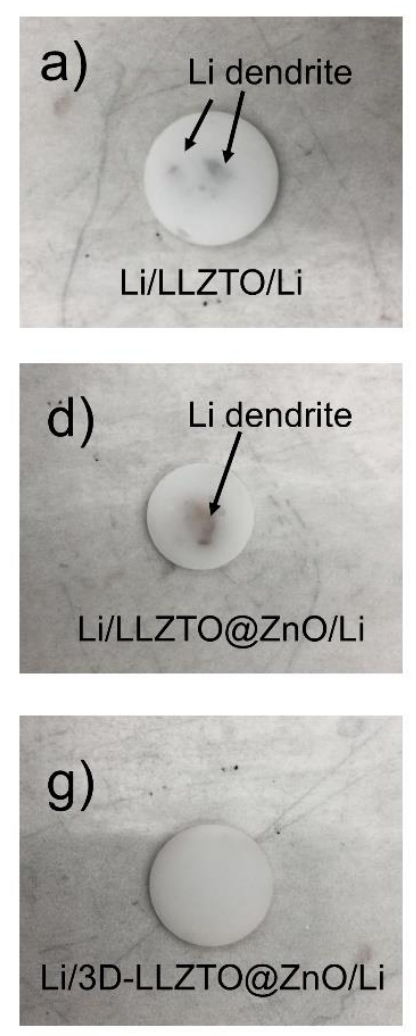
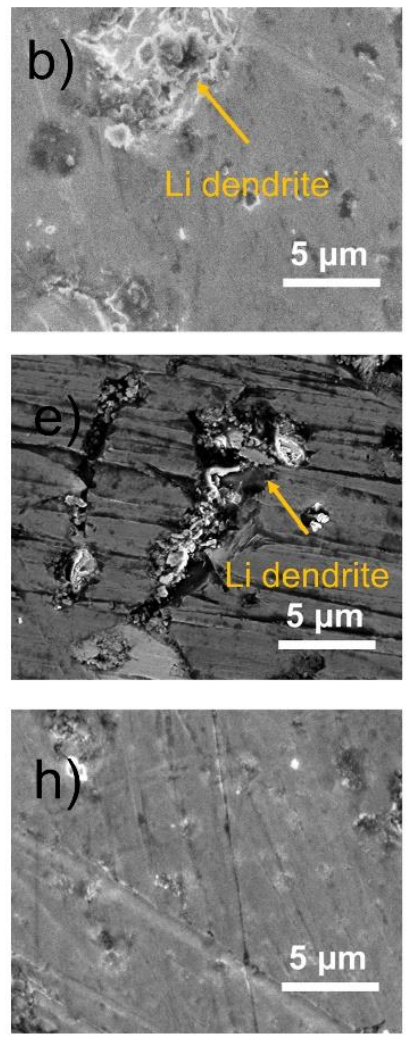
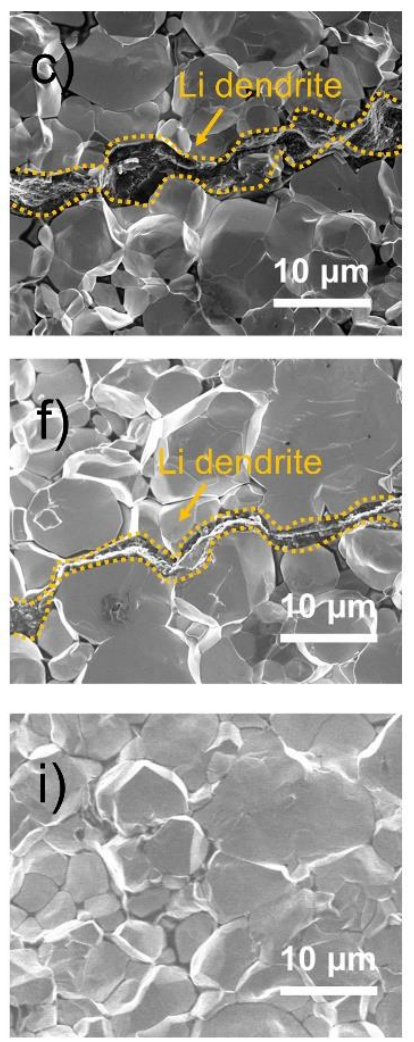

Figure S10 a) Optical image, b) surface morphology and c) cross-sectional SEM image of the Li/LLZTO/Li cell after short circuit at $0.5 \mathrm{~cm}^{-2}$ cycling. d) Optical image, e) surface morphology and f) cross-sectional SEM image of the Li/LLZTO@ZnO/Li cell after short circuit at $0.5 \mathrm{~cm}^{-2}$ cycling. g) Optical image, h) surface morphology and i) cross-sectional SEM image of the Li/3D-LLZTO@ZnO/Li cell after $600 \mathrm{~h}$ cycling at $0.5 \mathrm{~cm}^{-2}$ cycling. Note that the Li metal electrodes were removed by sanding. 

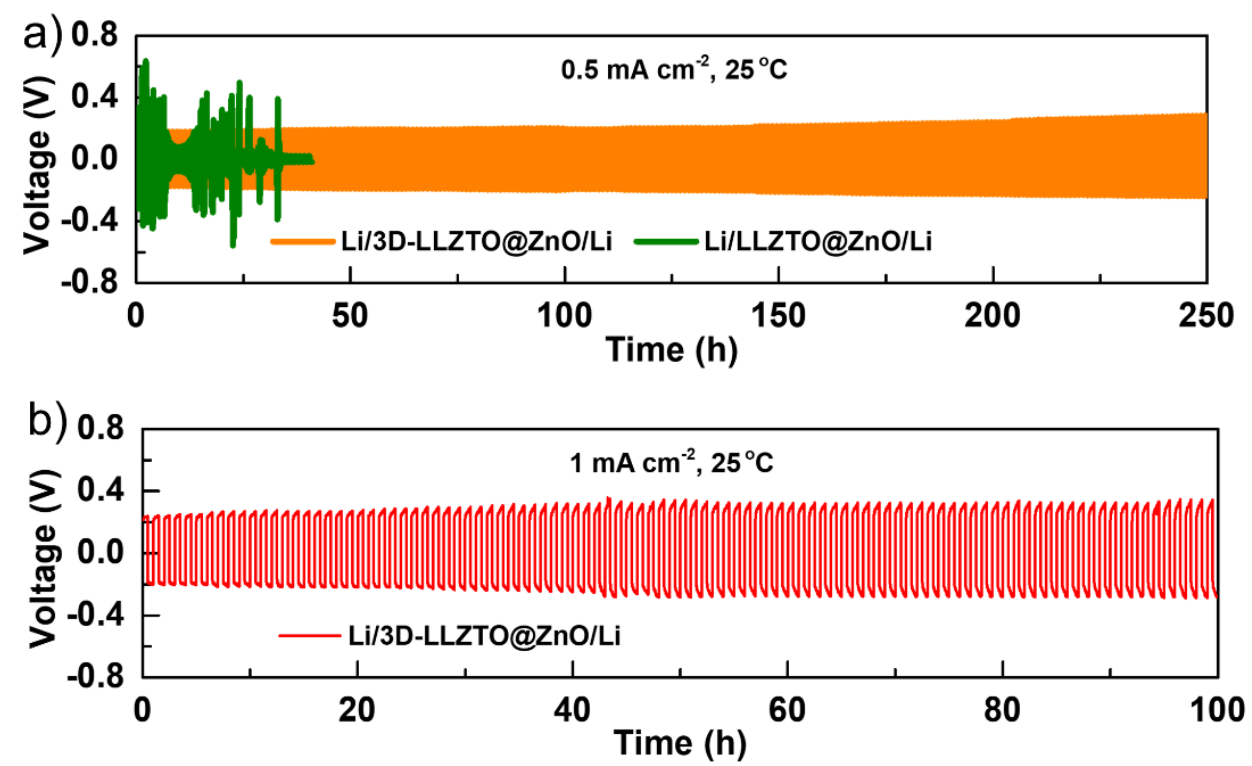

Figure S11 a) Galvanostatic cycling performance of the Li/LLZTO@ZnO/Li cell and Li/3D-LLZTO@ZnO/Li cell under $0.5 \mathrm{~mA} \mathrm{~cm}{ }^{-2}\left(0.25 \mathrm{mAh} \mathrm{cm}^{-2}\right)$ at $25{ }^{\circ} \mathrm{C}$. The thickness of Li anodes here is $\sim 30 \mu \mathrm{m}$. b) Galvanostatic cycling performance of the Li/3D-LLZTO@ZnO/Li cell at $1 \mathrm{~mA} \mathrm{~cm}^{-2}\left(0.5 \mathrm{mAh} \mathrm{cm}^{-2}\right)$. 


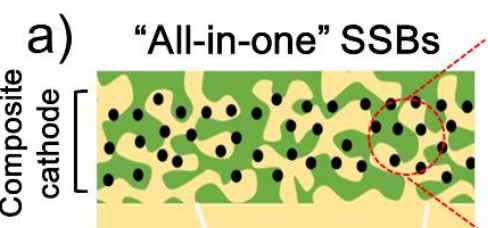

Acid-etched

LLZTO

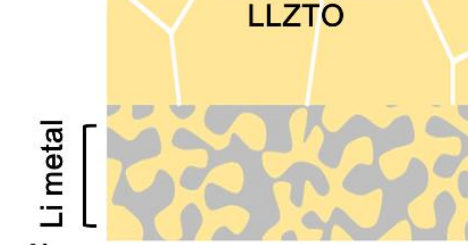

d)

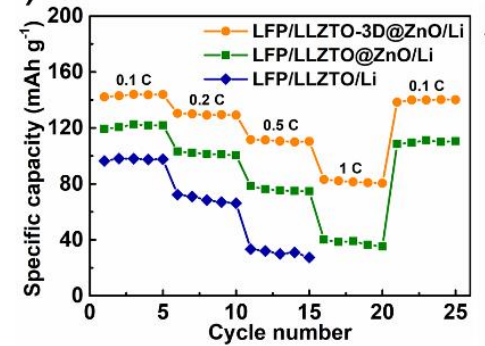

b)

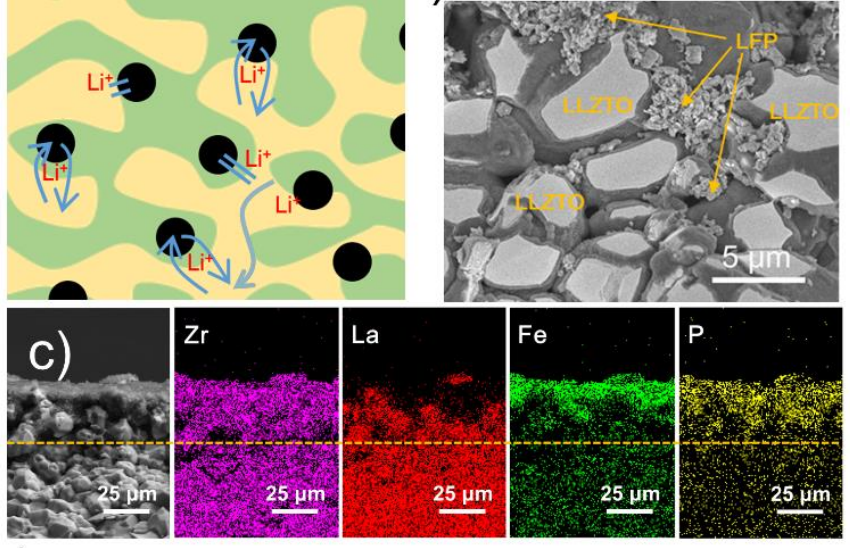

e)

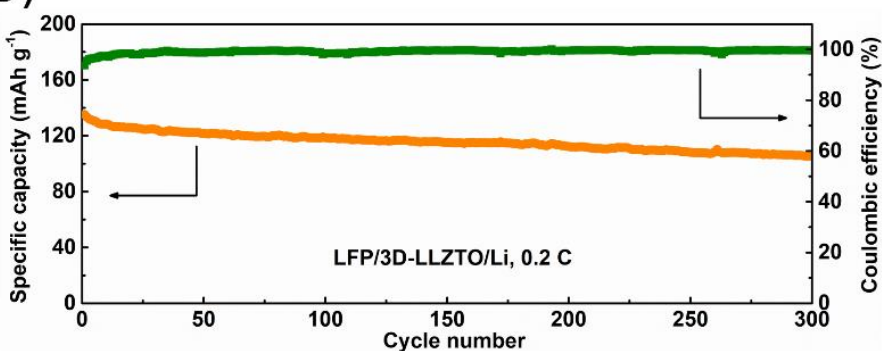

Figure S12 a) Schematic of "all-in-one" cells with a 3D Li anode and LFP cathode. b) cross-sectional SEM image and c) EDS mappings of the 3D LFP cathode. d) Rate performance of the LFP/LLZTO/Li, LFP/LLZTO@ZnO/Li and LFP/3DLLZTO@ZnO/Li cells. e) Cycle performance of the LFP/3D-LLZTO@ZnO/Li cell under $0.2 \mathrm{C}$ at $60{ }^{\circ} \mathrm{C}$. 

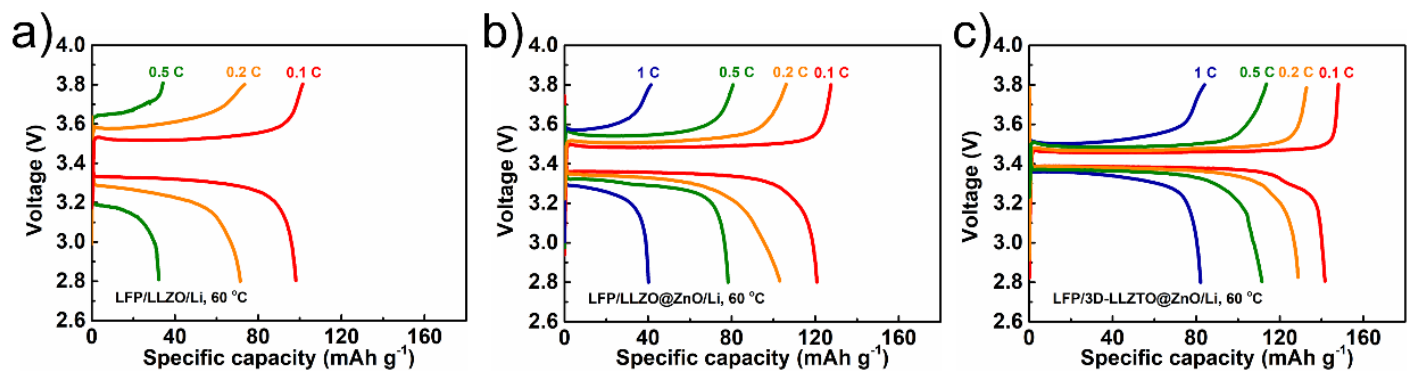

Figure S13 Charge/discharge curves of the LFP/LLZTO/LFP, LFP/LLZTO@ZnO/Li, and the LFP/3D-LLZTO@ZnO/Li cells at various current rates. 

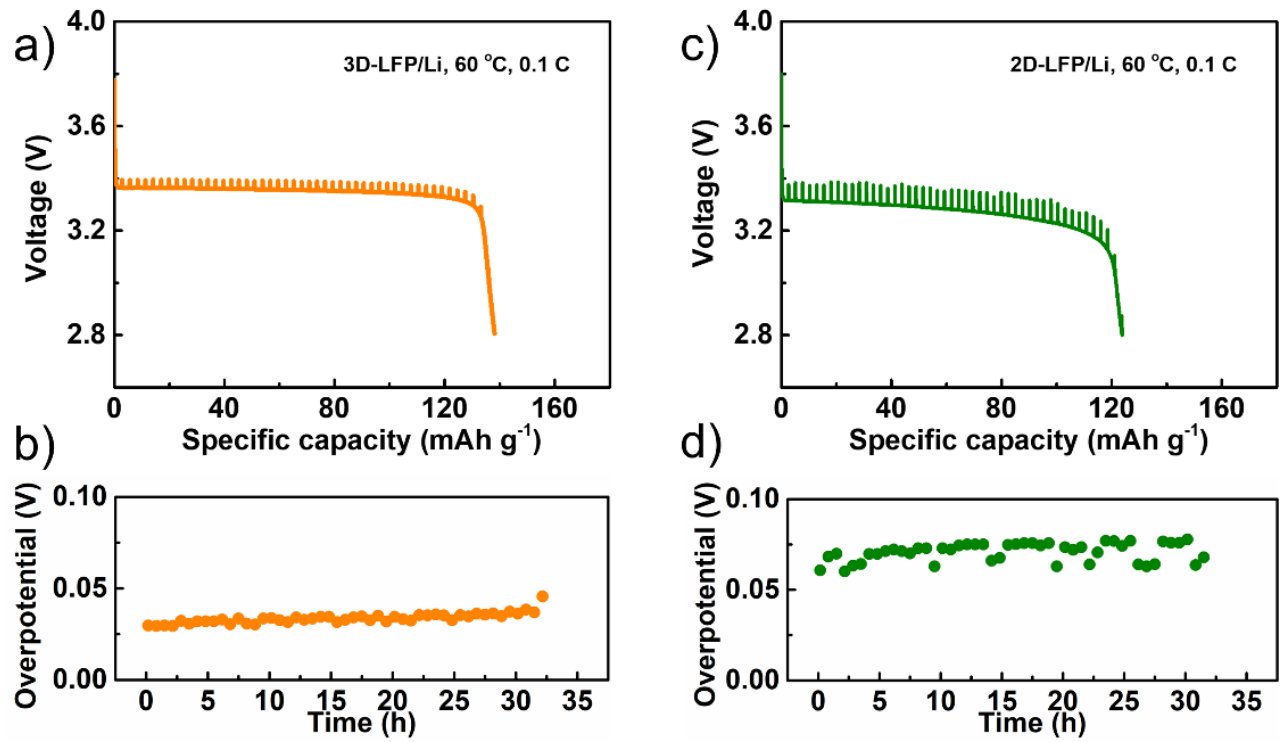

Figure S14 a) Transient discharge curves and b) polarization plots of 3D-LFP/Li cell under $0.1 \mathrm{C}$ at $60^{\circ} \mathrm{C}$ obtained by GITT. c) Transient discharge curves and d) polarization plots of 2D-LFP/Li cell under $0.1 \mathrm{C}$ at $60^{\circ} \mathrm{C}$ obtained by GITT.

\section{References}

(1) Du, F. M.; Zhao, N.; Li, Y. Q.; Chen, C.; Liu, Z. W.; Guo, X. X. All solid state lithium batteries based on lamellar garnet-type ceramic electrolytes. J. Power Sources 2015, 300, 24-28.

(2) Huo, H.; Zhao, N.; Sun, J.; Du, F.; Li, Y.; Guo, X. Composite electrolytes of polyethylene oxides/garnets interfacially wetted by ionic liquid for room-temperature solid-state lithium battery. $J$. Power Sources 2017, 372, 1-7.

(3) Pelton, A. The Li-Zn (lithium-zinc) system. J.phase equilibria 1991, 12 (1), 42-45.

(4) Wang, Z.; Zhang, H.; Wang, Z.; Zhang, L.; Yuan, J.; Yan, S.; Wang, C. Structure and strong ultraviolet emission characteristics of amorphous ZnO films grown by electrophoretic deposition. J. Mater. Res. 2003, 18 (1), 151-155. 